\title{
Aggregated Dynamic Equivalent of a Distribution System hosting Inverter-based Generators
}

\author{
Gilles Chaspierre \\ Dept. of Elec. Eng. and Comp. Science \\ University of Liège, Belgium \\ g.chaspierre@ulg.ac.be
}

\author{
Patrick Panciatici \\ Research \& Development Dept. \\ RTE, Versailles, France \\ patrick.panciatici@rte-france.com
}

\author{
Thierry Van Cutsem \\ Fund for Scientific Research (FNRS) \\ University of Liège, Belgium \\ t.vancutsem@ulg.ac.be
}

\begin{abstract}
An equivalent, i.e. a reduced-order model, of active distribution networks is derived, for use in (phasor-mode) dynamic simulations of large-disturbances. In the unreduced model, the network hosts a large number of inverter-based generators, responding to the disturbances in accordance with recent or nearfuture grid codes. The aggregated equivalent is of the "grey-box" type and its parameters are tuned in the least-square sense to match the dynamic responses of the unreduced system to several training disturbances. Changes in operating point are easily reflected when initializing the reduced model. Simulations are reported on a detailed 75-bus distribution system. The accuracy of the equivalent has been checked with respect to untrained disturbances and changes of the operating point.
\end{abstract}

Index Terms-Active distribution network, short-term dynamics, dynamic equivalent, inverter-based generation, dynamic load model.

\section{INTRODUCTION}

Distribution grids are expected to host an increasing amount of dispersed generation. Proper representation of Active Distribution Networks (ADNs) hosting numerous Inverter-Based Generators (IBGs) will thus become more and more important for Transmission System Operators (TSOs), when performing dynamic studies involving large disturbances in their grids. Using a single model involving both transmission and distribution systems is not only impractical, but would also entail data confidentiality issues. Equivalents are free from both problems since they are reduced-order, anonymized mathematical models. On the other hand, they must be accurate enough to be used in large-disturbance analysis.

Besides accuracy, several other features are desirable:

1) be able to reproduce discrete events and discontinuities taking place in IBGs subject to large disturbances;

2) be compatible with standard dynamic simulation software. The focus here is on simulation in phasor mode;

3) be physically intuitive as far as possible;

4) be easy to update when the operating point of the replaced distribution system changes, under the effect of load and variable renewable energy sources;

5) be valid in a wide range of operating conditions.

A classification of methods to derive ADN equivalents is given in e.g. [1] and [2].

A first category of approaches is based on system linearization and modal analysis. One example is provided by Ref. [3] where the Hankel norm approximation is used. Those approaches, unfortunately, are not likely to capture the nonlinear and discontinuous response of IBGs to large disturbances.

The "measurement-based" and "simulation-based" methods make up a second family, where the ADN responses are either measured or simulated, and model parameters are adjusted to fit those responses. Since high-severity events are rare, the measurement-based approaches lack information needed to identify models valid for large disturbances.

Among them, the "grey-box" approaches, as defined and recommended in [4] and [5], are attractive when sufficient physical knowledge is available. They rely on a reduced model that mimics the behaviour of the components it replaces, leading to a model with known structure but unknown parameters. A method of this category has been retained in this work, as it meets requirements 1) to 3) very well. The parameters are tuned at a given operating point. To meet requirements 4) and 5 ), changes in the operating point are reflected in the initial value of the model state variables, while the parameters are kept unchanged.

Examples of "grey-box" generic ADN models are presented in [6], validated in [7] and further improved in [8].

Yet it is not clear whether existing grey-box equivalent models are able to account for the discrete events triggered by inverter controls in response to large disturbances. The model proposed in this paper tackles this issue. It is a significant extension of the one in [9], in terms of model structure, state initialization and parameter tuning.

The rest of the paper is organized as follows. The identification of the equivalent is detailed in Section II. In Section III, the load and IBG models are described. The unreduced system is presented in Section IV while the simulation results are given in Section V. The main achievements and future investigations are summarized in Section VI.

\section{IDENTIFICATION OF THE ADN EQUIVALENT}

\section{A. Structure of the ADN equivalent}

The structure of the equivalent is shown in the left part of Fig. 1. Its main feature is the aggregation of the originally distributed IBGs (resp. loads) into two lumped, equivalent IBGs (resp. loads). The latter are differentiated by voltage levels, to separate medium and low voltage equipments, respectively. The aggregated components are placed behind 


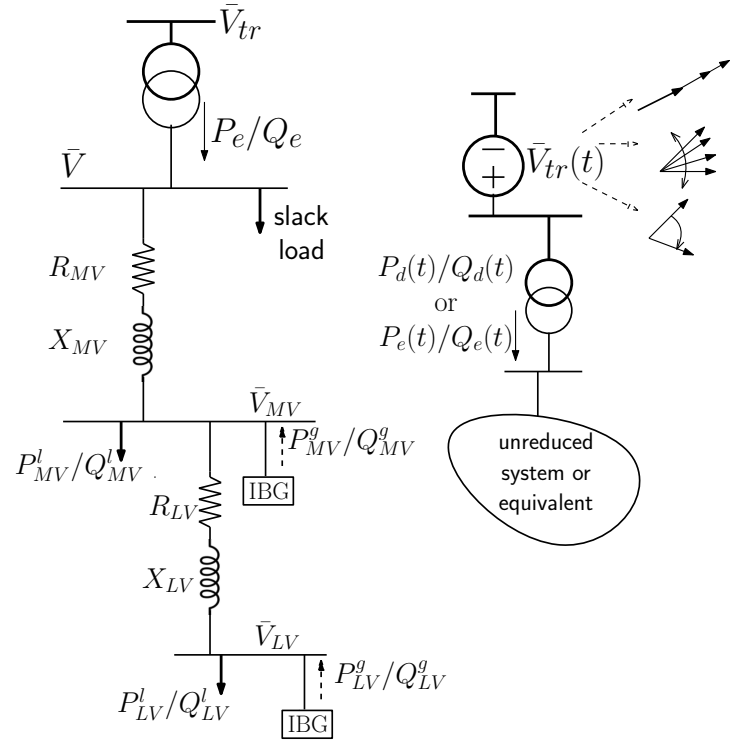

Figure 1. Structure of the equivalent and training signals

equivalent impedances accounting for the network effects. The main substation transformer is modeled explicitly.

\section{B. Optimizing the ADN equivalent parameters}

Unlike equivalents derived from linearized models, an equivalent valid for large disturbances should be derived from multiple "training" scenarios involving representative disturbances. The scenarios are obtained by replacing the transmission system with a time-varying voltage source $\bar{V}_{t r}(t)$, as shown in the right part of Fig. 1. The $\bar{V}_{t r}(t)$ signals should involve typical variations of the corresponding voltage, such as variations of amplitude, phase jumps, change of frequency, and their combinations.

The $\bar{V}_{t r}(t)$ signals are applied to both the unreduced ADN model and the equivalent. The outputs of interest are the active and reactive powers entering the distribution grid.

The parameters of the equivalent are grouped in the $\boldsymbol{\theta}$ vector.

Let $m$ be the number of training signals. For the $j$-th signal $(j=1, \ldots, m)$, let us denote by:

$P_{e}(\boldsymbol{\theta}, j, k)$ the discrete-time evolution of the active power entering the equivalent system;

$Q_{e}(\boldsymbol{\theta}, j, k)$ the corresponding evolution of reactive power;

$P_{d}(j, k) \quad$ the discrete-time evolution of the active power entering the unreduced system;

$Q_{d}(j, k) \quad$ the corresponding evolution of reactive power,

where $k$ refers to the discrete times used by the timesimulation solver. The same time instants are considered for both the unreduced and the equivalent system; if needed, interpolation is used to make the time instants coincide. The number of discrete times is denoted by $n$.

$\boldsymbol{\theta}$ is adjusted to match the $m$ dynamic responses of the unreduced system all together in the least-square sense. This consists of minimizing:

$$
\varepsilon(\boldsymbol{\theta})=\varepsilon_{P}(\boldsymbol{\theta})+w \varepsilon_{Q}(\boldsymbol{\theta})
$$

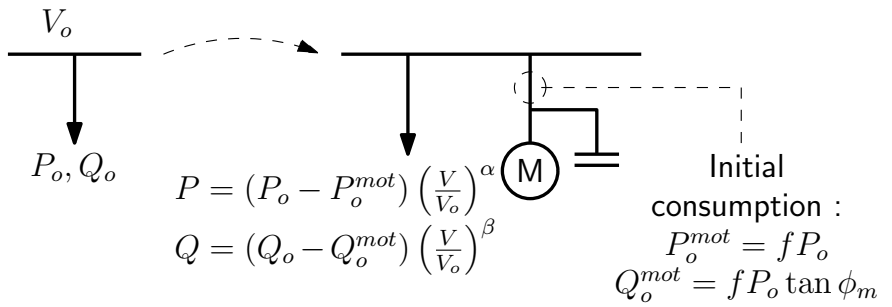

Figure 2. Load composition. $P_{o}$ (resp. $Q_{o}$ ) stands for either $P_{M V}^{\ell}$ or $P_{L V}^{\ell}$, and $Q_{o}$ for either $Q_{M V}^{\ell}$ or $Q_{L V}^{\ell}$

where:

$$
\begin{aligned}
\varepsilon_{P}(\boldsymbol{\theta}) & =\sum_{j=1}^{m} \frac{1}{n} \sum_{k=1}^{n}\left[P_{e}(\boldsymbol{\theta}, j, k)-P_{d}(j, k)\right]^{2} \\
\varepsilon_{Q}(\boldsymbol{\theta}) & =\sum_{j=1}^{m} \frac{1}{n} \sum_{k=1}^{n}\left[Q_{e}(\boldsymbol{\theta}, j, k)-Q_{d}(j, k)\right]^{2}
\end{aligned}
$$

under the constraints: $\boldsymbol{\theta}^{L} \leq \boldsymbol{\theta} \leq \boldsymbol{\theta}^{U}$. $w$ is the weight assigned to the reactive power responses with respect to the active ones. The bounds $\boldsymbol{\theta}^{L}$ and $\boldsymbol{\theta}^{U}$ keep $\boldsymbol{\theta}$ in realistic ranges of values, and are used to generate an initial guess of $\boldsymbol{\theta}$.

An analytical expression of the first- and second-order derivatives of $\varepsilon$ with respect to $\theta$ cannot be derived. Hence, standard least-square methods cannot be used unless those derivatives are estimated numerically. At this stage, a metaheuristic optimization method has been preferred, more precisely a variant of the Differential Evolution algorithm [10]. Please refer to [9] for additional information.

\section{Initialization of equivalent}

With reference to Fig. 1, the initial active and reactive powers $P_{M V}^{\ell}$ and $Q_{M V}^{\ell}$ (resp. $P_{M V}^{g}$ and $Q_{M V}^{g}$ ) are obtained by aggregating the consumptions of all dispersed loads (resp. the productions of all dispersed IBGs) that are connected at MV buses in the unreduced model. The same applies to loads and IBGs connected at LV level and aggregated at the LV equivalent bus, yielding $P_{L V}^{\ell}, Q_{L V}^{\ell}, P_{L V}^{g}$ and $Q_{L V}^{g}$.

1) When identifying the equivalent: the operating point is fixed. However, the equivalent resistances $R_{M V}, R_{L V}$ and reactances $X_{M V}, X_{L V}$, being components of $\boldsymbol{\theta}$, are varied during the minimization of $\varepsilon(\boldsymbol{\theta})$. Hence, for each value of $\boldsymbol{\theta}$, a simple 3-bus power flow problem is solved to determine the initial voltages $\bar{V}_{L V}$ and $\bar{V}_{M V}$. The initial voltage $\bar{V}$ at the MV bus of the transformer (see Fig. 1) is fixed.

The losses in the two equivalent impedances change from one value of $\boldsymbol{\theta}$ to another. On the other, the active and reactive powers injected into the distribution grid must remain unchanged. Hence, to satisfy the power balance, a "slack" load is added, as shown in Fig. 1. This load is usually small.

2) When using the equivalent at a different operating point: its parameters $\boldsymbol{\theta}$ remain constant but the powers $P_{M V}^{\ell}, Q_{M V}^{\ell}, \ldots, P_{L V}^{g}, Q_{L V}^{g}$ are updated as described above. The same 3-bus power flow problem is solved. The slack load is also needed to match the power flows $P_{e}, Q_{e}$ see from the transmission side. 


\section{LOAD AND IBG GENERIC MODELS}

\section{A. Load model}

As shown in Fig. 2, each load is split into an equivalent 3rd-order induction motor (taking initially a fraction $f$ of the load active power) and a static part with exponential model.

\section{B. IBG model}

The IBG model is given in block-diagram form in Fig. 3. Instead of focusing on internal components, the model aims at reproducing the IBG response to terminal voltage changes in accordance with most grid codes [11], [12], [13].

$v_{x}$ and $v_{y}$ (resp. $i_{x}$ and $i_{y}$ ) are the projections on rotating reference axes $(x, y)$ of the phasor of the terminal voltage (resp. the current injected into the grid). All blocks rely on the measured terminal voltage $V_{m}$ obtained from the terminal voltage with the time constant $T_{m}$. The equivalent time constant $T_{g}$ accounts for the power electronics.

Other parts of the model are described hereafter. Please refer to [9] for more details.

1) Phase Locked Loop (PLL): The PLL dynamics is represented in some detail. The gain $k_{P L L}$ determines its response time. $V_{P L L}$ is the voltage threshold below which it is blocked.

2) Low Voltage Ride-Through (LVRT): LVRT capability is an important feature of IBGs, requiring them to remain connected to the grid during a disturbance as long as the voltage is above a reference curve, as shown in Fig. 4.

3) Reactive current injection: In low voltage conditions IBGs are requested to inject reactive current to support their terminal voltages. The injected current varies linearly with the measured voltage magnitude as shown in Fig. 5.

4) Active power recovery: When the IBG is called to support the grid voltage, it may happen that the active current is reduced to leave room for its reactive counterpart without exceeding the inverter current limit. Once the voltage has recovered to normal values, the IBG recovers its active current. This cannot take place too rapidly but it should not take too much time either, to avoid a power imbalance. Some grid codes specify a range of allowed values for the rate of increase of the active current (e.g. [13]).

5) Partial tripping: The equivalent IBG must account for the tripping of some of the individual IBGs it replaces. The main issue is that the equivalent has a single terminal voltage while the individual IBG voltages differ from one bus to another throughout the distribution network. The problem has been tackled by providing the equivalent IBGs with a "partial tripping" feature. The latter consists of multiplying the output current by a factor $f_{1} f_{2} f_{3}$ with $0 \leq f_{1}, f_{2}, f_{3} \leq 1$, where $f_{1}, f_{2}$ and $f_{3}$ relate respectively to the time intervals $\left[0 T_{1}\right]$, $\left[T_{1} T_{i n t}\right]$ and $\left[T_{i n t} T_{2}\right]$ of the LVRT curve in Fig. 4 [9].

\section{Parameters to identify}

The resulting $\boldsymbol{\theta}$ vector has 40 components. This includes:

- for the IBGs: the nominal apparent power, the time constant $T_{g}$ (see Fig. 3), parameters of the LVRT curve (see Fig. 4), the $k_{R C I}$ slope and the $V_{S 1}$ threshold (see Fig. 5), the rate of recovery of the active current, parameters involved in the partial tripping;

- for the loads: the fraction $f$, the exponents $\alpha, \beta$, the initial power factor and 8 parameters of the motor (see Fig. 2);

- for the network: $R_{M V}, X_{M V}, R_{L V}, X_{L V}$ (see Fig. 1).

\section{THE UNREDUCED SYSTEM}

\section{A. Modelling and data}

The 75-bus 11-kV distribution grid previously considered in [14] has been used in this study. Its one-line diagram is shown in Fig. 6. The network feeds 75 loads and hosts 75 IBGs.

Among the $75 \mathrm{MV}$ buses, 38 feed Low-Voltage (LV) distribution grids hosting small residential IBGs (essentially photovoltaic units). The corresponding load is $4.7 \mathrm{MW}$ and the production is $1.9 \mathrm{MW}$. Each MV bus injection is modeled as shown in Fig. 7.a with a lumped load and a lumped IBG unit behind an impedance $R_{e}+j X_{e}$ accounting for the MV/LV transformer and the $\mathrm{LV}$ feeder(s). At the remaining $37 \mathrm{MV}$ buses, the injection is modeled by an industrial load and a large IBG unit behind a transformer, as shown in Fig. 7.b. These IBGs have fault-ride-through and reactive current injection capabilities, while the ones in the LV grids do not. The parameters of the respective fault-ride-through characteristics are given in Fig. 4. The corresponding load is $24.7 \mathrm{MW}$ and the production is $19.2 \mathrm{MW}$.

It must be emphasized that the IBG and load data have been randomized from one bus to another. The so varied parameters are those listed in Section III-C as well as $R_{e}, X_{e}$ (see Fig. 7.a). The time constant $T_{m}$ has been set to 20 (resp. 30) $\mathrm{ms}$ for large (resp. small) IBG units. Assuming that IBGs obey the rules in force in the ADN, the LVRT characteristic has not been randomized. As regards the reactive current injection characteristic, grid codes usually specify a range of values for the slope $k_{R C I}$ (e.g. [12]) and, hence, this parameter has been randomized, while $m$ and $V_{S 1}$ have not.

The unreduced model involves 7,650 differential-algebraic equations vs. only 215 equations for the equivalent.

\section{B. Example of IBG response to a fault}

An example of IBG response to a fault, that the equivalent must reproduce at a larger scale, is given hereafter.

A short-circuit taking place at $t=0.1 \mathrm{~s}$ and lasting for $100 \mathrm{~ms}$ has been applied at some distance of a large IBG unit. Figure 8 shows the evolution of the active and reactive currents injected in the grid. The unit operates initially at unity power factor. In accordance with the grid code, it injects reactive current during the fault in order to support its terminal voltage, while the active current is notably reduced in order not exceed the maximum current of the inverter. It takes $1.5 \mathrm{~s}$ for the active current to recover its pre-disturbance value.

\section{Simulation Results}

The results aim at comparing the response to large disturbances of the powers entering the distribution grid (see Fig. 1), in the unreduced and the equivalent models, respectively.

The time simulations have been performed with the RAMSES software developed at the Univ. of Liège [15]. 


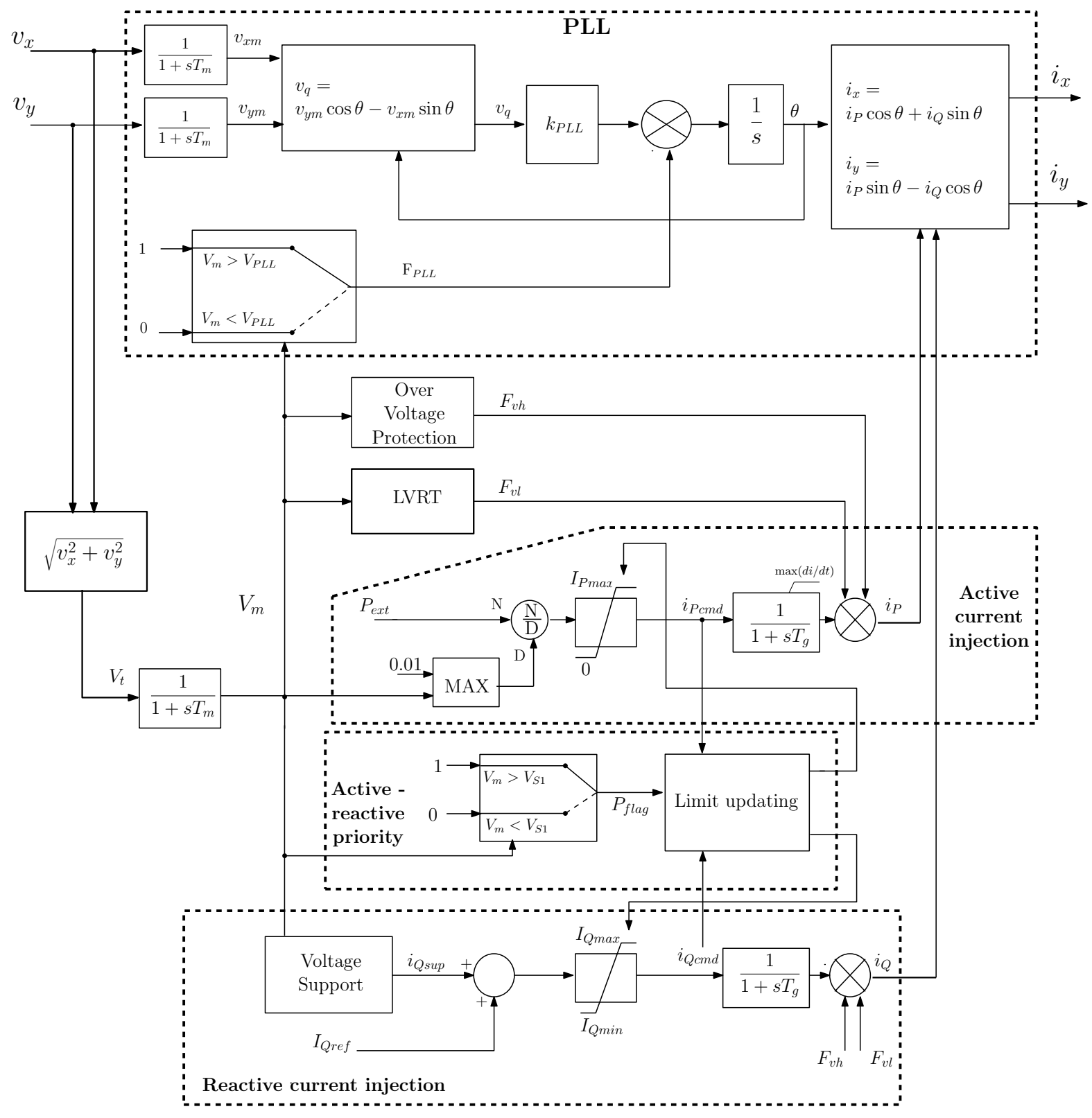

Figure 3. IBG generic model

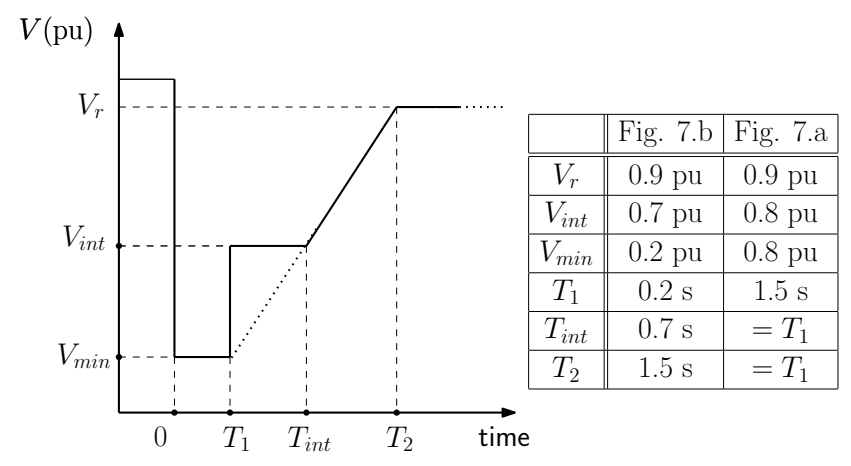

Figure 4. LVRT characteristic (fault occurring at $t=0$ )

\section{A. Training of the ADN equivalent}

The training signals considered in this paper are transmission voltage dips, i.e. changes in magnitude of $\bar{V}_{t r}$ (see Fig. 1).

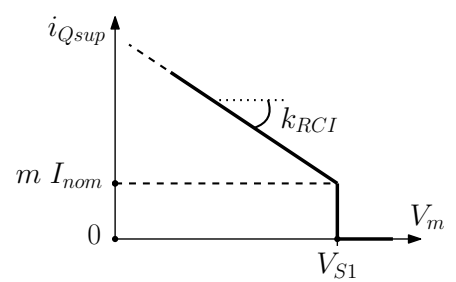

Figure 5. Reactive current injection characteristic. $i_{Q s u p}$ appears in Fig. 3

To simulate faults of various severities, the duration and the depth of the dip has been varied as detailed in Fig. 9. $\boldsymbol{\theta}$ has been adjusted to match the corresponding $m=10$ responses.

For example, the results relative to the training signal No. 7 are shown in Figs. 10 and 11. The effect of the reactive current injected during the fault by the IBGs at MV level can be seen in Fig. 11, with the power flow changing from 


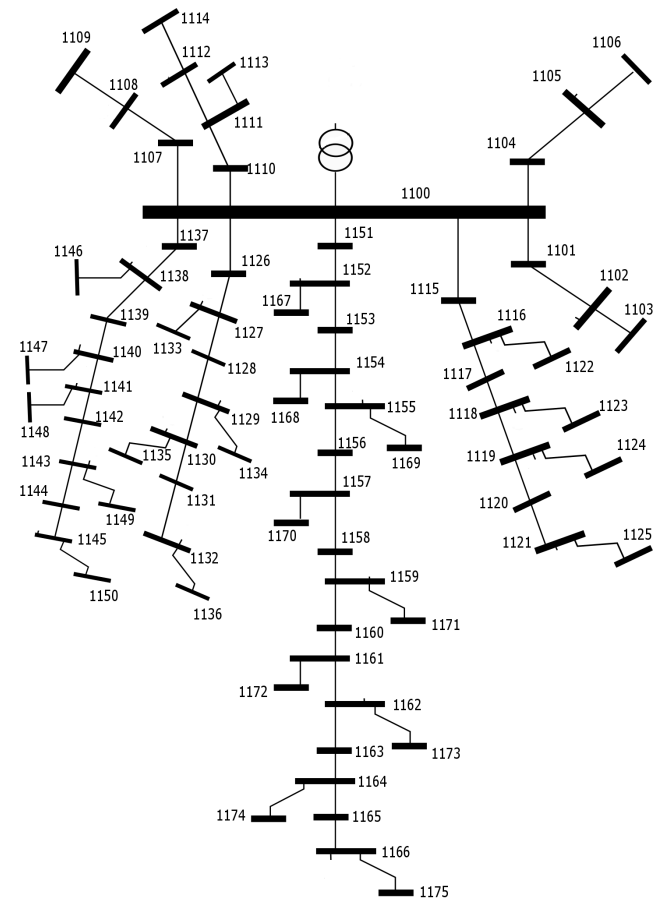

Figure 6. One-line diagram of the 75 -bus $11-\mathrm{kV}$ distribution system

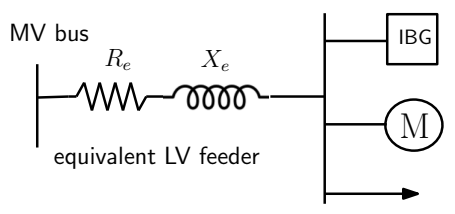

b.

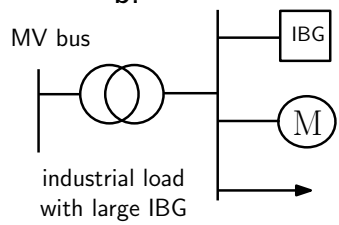

Figure 7. MV bus injection model: (a) equivalent LV feeder; (b) industrial load combined with a large IBG unit

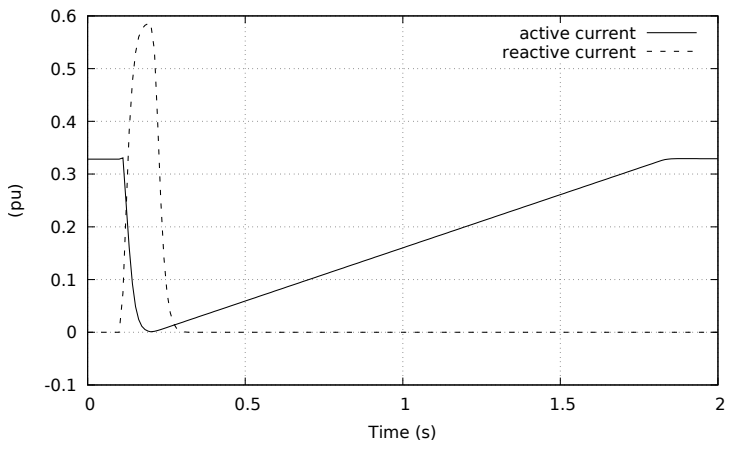

Figure 8. Active and reactive currents of an IBG in response to a fault

import to export. The most striking fact is the sharp increase of active and reactive powers immediately after fault clearing. The reason is twofold. First, the motors draw additional power when re-accelerating. Second, during the fault, the active currents of IBGs at MV level have been reduced, if not canceled, owing to the priority given to reactive currents for voltage support. The restoration of IBG active powers takes between one and two seconds and its effect is seen in the progressive reduction of the imported active power. Finally, it is noted that active power does not return to its pre-disturbance value. One reason is the tripping of the units connected to $\mathrm{LV}$

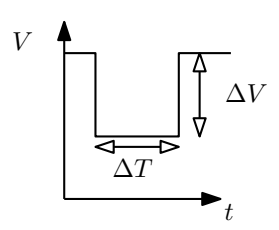

\begin{tabular}{|c|c|c|}
\hline Signal No & $\Delta V(\mathrm{pu})$ & $\Delta T(\mathrm{~s})$ \\
\hline \hline 1 & 0.5 & 0.10 \\
\hline 2 & 0.5 & 0.25 \\
\hline 3 & 0.6 & 0.10 \\
\hline 4 & 0.6 & 0.25 \\
\hline 5 & 0.7 & 0.10 \\
\hline 6 & 0.7 & 0.25 \\
\hline 7 & 0.8 & 0.10 \\
\hline 8 & 0.8 & 0.25 \\
\hline 9 & 0.9 & 0.10 \\
\hline 10 & 0.9 & 0.25 \\
\hline
\end{tabular}

Figure 9. Signals considered in the training phase

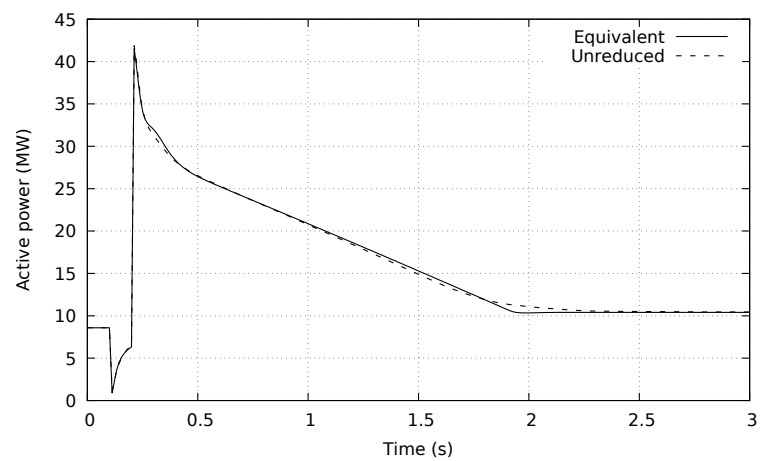

Figure 10. Training signal No. 7: Comparison of active power responses of the unreduced and equivalent models

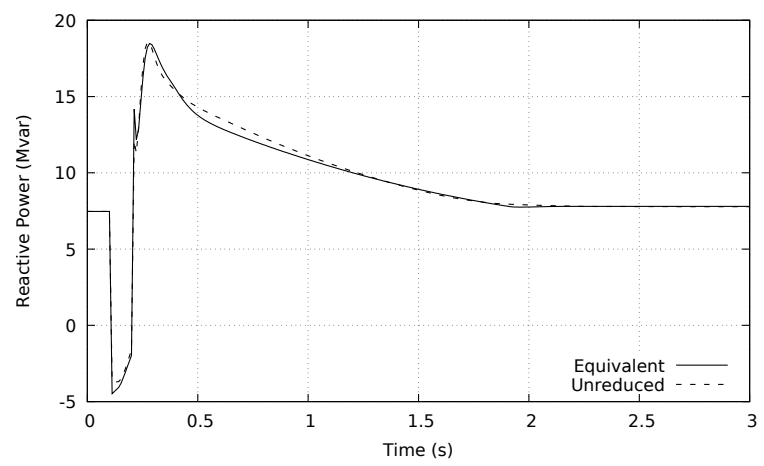

Figure 11. Training signal No. 7: Comparison of reactive power responses of the unreduced and equivalent models

grids, allowed by their more permissive LVRT characteristic (see Fig. 4). Another reason is the randomization of the $k_{R C I}$ parameter (see Fig. 5) resulting in smaller voltage support by some IBGs at MV level and, hence, a higher probability for the voltage to cross the LVRT characteristic.

The adjusted parameters yield, in all 10 cases, a response of the equivalent very close to that of the unreduced system. In particular, the final values are identical, indicating a correct estimation of the number of IBGs tripped.

\section{B. Accuracy of the ADN equivalent in non trained conditions}

It is important to check the accuracy of the equivalent facing other signals than those used for training. The results presented in the rest of the paper were obtained when varying the magnitude of $\bar{V}_{t r}$ as shown in Fig. 12, representative of 


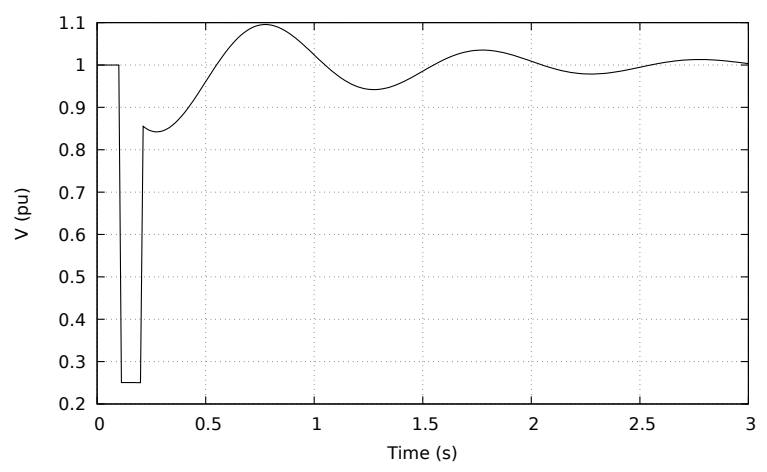

Figure 12. Voltage disturbance used for ADN equivalent validation

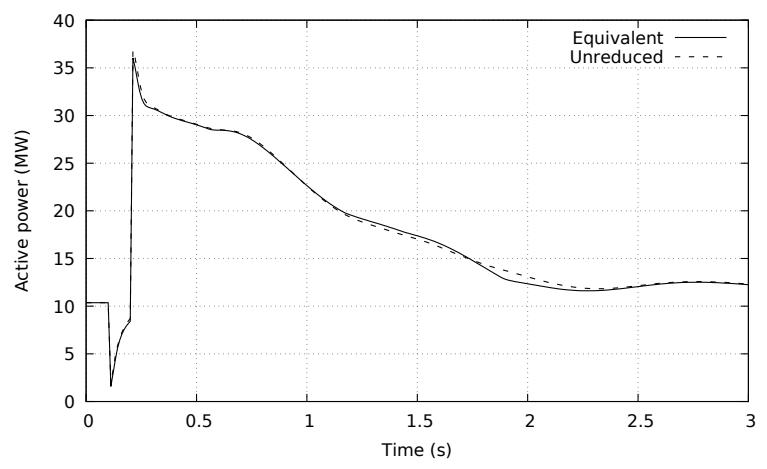

Figure 13. Modified operating point 1). Comparison of active power responses of the unreduced and equivalent models

electromechanical oscillations in the transmission system. Yet the phase angle and the frequency of $\bar{V}_{t r}$ are constant.

Furthermore, as mentioned in the Introduction, the equivalent should be valid in a wide enough range of operating conditions of the ADN. Two tests at different operating points are reported hereafter:

1) the initial power consumed by the loads in the unreduced system was increased by $20 \%$ : the responses to the disturbance of Fig. 12 are given in Figs. 13 and 14;

2) the initial active power production of IBGs was reduced by $50 \%$ while the load was decreased by the same amount, leaving the net power inflow in the ADN unchanged. The responses to the disturbance of Fig. 12 are given in Figs. 15 and 16.

In all cases, the equivalent is initialized as described in Section II-C2. Let us recall that $\boldsymbol{\theta}$ is not updated.

It can be seen that the equivalent approaches the original system very closely.

\section{Robustness with respect to modelling errors}

The equivalent is a low-order approximation of a reference model, which should be set up as accurately as possible. However, in practice, there is uncertainty on the load and even the IBG models and parameters. The test reported hereafter consists of examining the accuracy of the equivalent with respect to unreduced models when the latter somewhat differ from what was assumed to derive the equivalent.

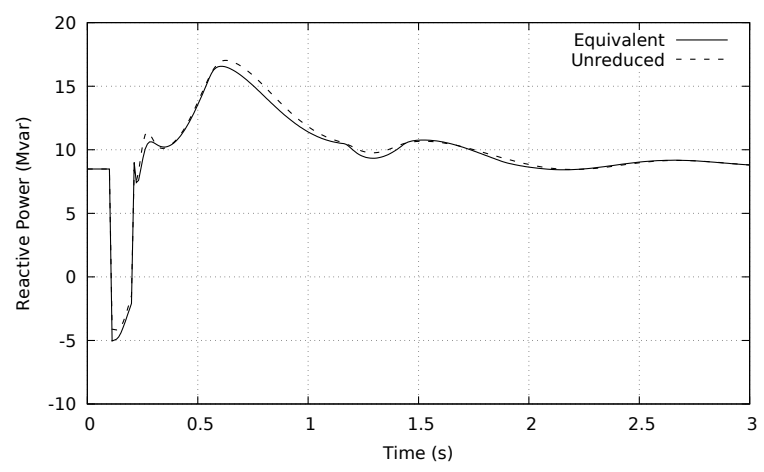

Figure 14. Modified operating point 1). Comparison of reactive power responses of the unreduced and equivalent models

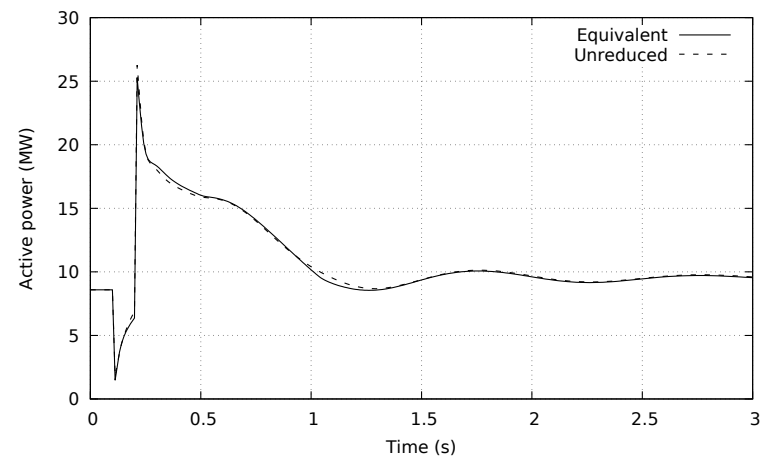

Figure 15. Modified operating point 2). Comparison of active power responses of the unreduced and equivalent models

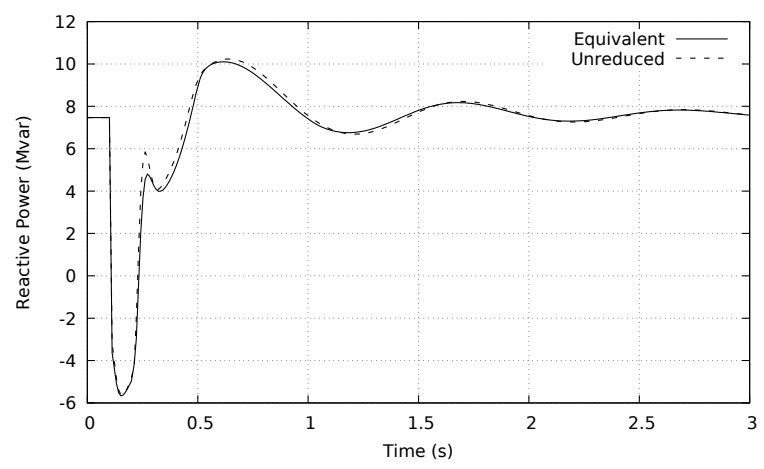

Figure 16. Modified operating point 2). Comparison of reactive power responses of the unreduced and equivalent models

The initial load and IBG powers are unchanged, but the proportion of motor load (see Fig. 7) has been increased at each bus by $10 \%$ and $20 \%$, respectively. A lower accuracy is to be expected, since the effective proportion is unknown and the equivalent cannot be updated as it was for known changes of the operating point. The active and reactive power responses are given in Figs. 17 and 18, respectively. Compared to the previous tests, they show a larger discrepancy between the equivalent and the unreduced models, essentially over a time interval of $0.7 \mathrm{~s}$ after fault clearing, when motors re-accelerate. However, for the assumed model inaccuracy, the discrepancy 


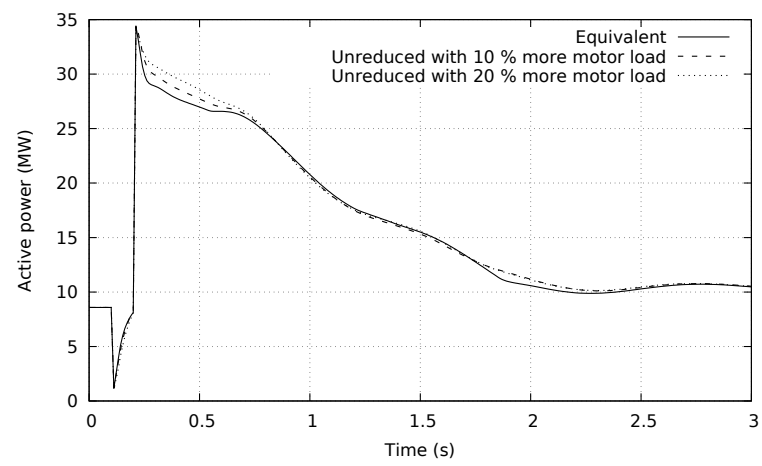

Figure 17. Comparison of active power responses of the equivalent and unreduced models corresponding to two proportions of motor load

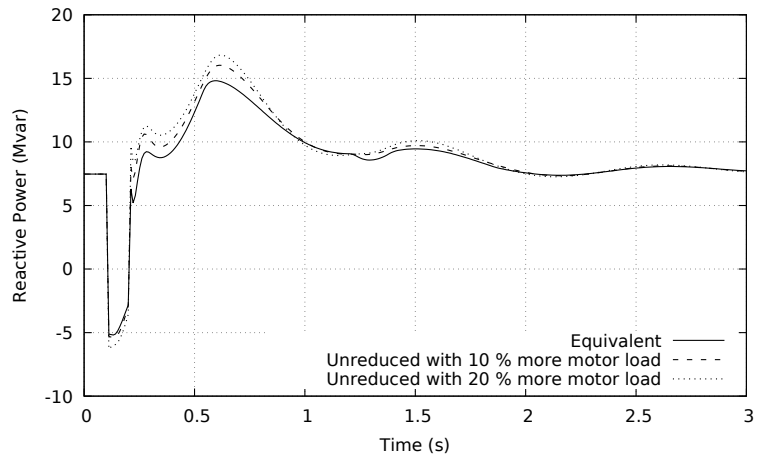

Figure 18. Comparison of reactive power responses of the equivalent and unreduced models corresponding to two proportions of motor load

remains acceptable.

\section{SUMMARY AND PERSPECTIVES}

The on-going research reported in this paper aims at deriving a dynamic equivalent of a distribution network hosting numerous IBGs, for use in dynamic simulations of large disturbances, at transmission level. The size of the original model is significantly reduced by aggregating the IBGs following the same grid code requirements into an equivalent IBG, mimicking their common behaviour, in particular mode switchings and other nonlinearities. Loads are aggregated similarly. The equivalent is easily updated with changing operating conditions.

The parameters of the equivalent are determined to match a number of training responses in the least-square sense, thereby avoiding overfitting a particular scenario.

Encouraging simulation results have been found as regards the accuracy of the equivalent facing disturbances not involved in the training phase and/or after changes in the initial operating point of the distribution system.

The following extensions are currently being investigated :

- the parameters of the equivalent are adjusted, and its accuracy is being tested in the presence of more complex variations of the transmission voltage, involving for instance phase jumps or frequency changes;

- further validation tests are carried out with simulated measurement noise;
- currently, the reduced model involves as many as 40 parameters. It is of interest to identify which ones have the most influence, and possibly exclude the others from the optimization. Besides a higher computational efficiency (allowing more frequent parameter updates), the objective is to make the equivalent easier to interpret by end-users;

- so far, a meta-heuristic global optimization method has been used for least-square minimization. An alternative "derivative-free" optimization method is sought, with better control of the iterative procedure, fewer randomly generated parameter values, and higher computational efficiency.

\section{REFERENCES}

[1] U. D. Annakkage, N. K. C. Nair, Y. Liang, A. M. Gole, V. Dinavahi, B. Gustavsen, T. Noda, H. Ghasemi, A. Monti, M. Matar, R. Iravani, and J. A. Martinez, "Dynamic system equivalents: A survey of available techniques," IEEE Trans. on Power Delivery, vol. 27, no. 1, pp. 411-420, 2012.

[2] F. O. Resende, J. Matevosyan, and J. V. Milanovic, "Application of dynamic equivalence techniques to derive aggregated models of active distribution network cells and microgrids," Proc. 2013 IEEE PES Grenoble PowerTech conf., pp. 1-6, 2013.

[3] A. Ishchenko, J. Myrzik, and W. Kling, "Dynamic equivalencing of distribution networks with dispersed generation using hankel norm approximation," IET Generation, Transmission \& Distribution, vol. 1, no. 5, pp. 818-825, 2007.

[4] Milanović, Jovica (convener), "Modelling and aggregation of loads in flexible power networks," Report of CIGRE WG C4.605, 2014.

[5] Hatziargyriou, Nikos (convener), "Contribution to bulk system control and stability by distributed energy resources connected at distribution network," IEEE PES Tech. Report PES-TR22, 2017.

[6] S. M. Zali and J. V. Milanovic, "Generic Model of Active Distribution Network for Large Power System Stability Studies," IEEE Trans. on Power Systems, vol. 28, no. 3, pp. 3126-3133, 2013.

[7] J. V. Milanovic and S. Mat Zali, "Validation of equivalent dynamic model of active distribution network cell," IEEE Trans. on Power Systems, vol. 28, no. 3, pp. 2101-2110, 2013.

[8] F. Conte, F. D. Agostino, S. Massucco, G. Palombo, F. Silvestro, S. Energetico, and S. Rse, "Dynamic Equivalent Modelling of Active Distribution Networks for TSO-DSO Interactions," Proc. IEEE ISGT Europe conference, Torino (Italy), 2017, in press.

[9] G. Chaspierre, P. Panciatici, and T. Van Cutsem, "Dynamic Equivalent of a Distribution Grid Hosting Dispersed Photovoltaic Units,' Proc. IREP' 17 Symposium, Espinho (Portugal), 2017, in press.

[10] K. Price, R. M. Storn, and J. A. Lampinen, Differential Evolution - A Practical Approach to Global Optimization. Springer, 2005.

[11] P. Kotsampopoulos, N. Hatziargyriou, B. Bletterie, and G. Lauss, "Review, analysis and recommendations on recent guidelines for the provision of ancillary services by Distributed Generation," Proc. IEEE Intern. Workshop on Intelligent Energy Systems, pp. 185-190, 2013.

[12] VDE-AR-N 4120, "Technical requirements for the connection and operation of customer installations to the high-voltage network (TCC High-Voltage)," pp. 1-123, January 2015.

[13] B. Weise, "Impact of k-factor and active current reduction during fault-ride-through of generating units connected via voltage-sourced converters on power system stability," IET Renewable Power Generation, vol. 9, no. 1, pp. 25-36, 2015.

[14] H. Soleimani Bidgoli, M. Glavic, and T. Van Cutsem, "RecedingHorizon Control of Distributed Generation to Correct Voltage or Thermal Violations and Track Desired Schedules," Proc. 19th PSCC conf., 2016.

[15] P. Aristidou, D. Fabozzi, and T. Van Cutsem, "Dynamic simulation of large-scale power systems using a parallel Schur-complement-based decomposition method," IEEE Trans. on Parallel and Distributed Systems, vol. 25, no. 10, pp. 2561-2570, 2014. 